Supporting Information for

\title{
Impregnation of Cellulose Fibers with Copper Colloids and Their Processing into Electrically Conductive Paper
}

Murielle Schreck, Rupali Deshmukh, Elena Tervoort, Markus Niederberger*

Laboratory for Multifunctional Materials, Department of Materials, ETH Zurich,

Vladimir-Prelog-Weg 5, 8093 Zurich, Switzerland

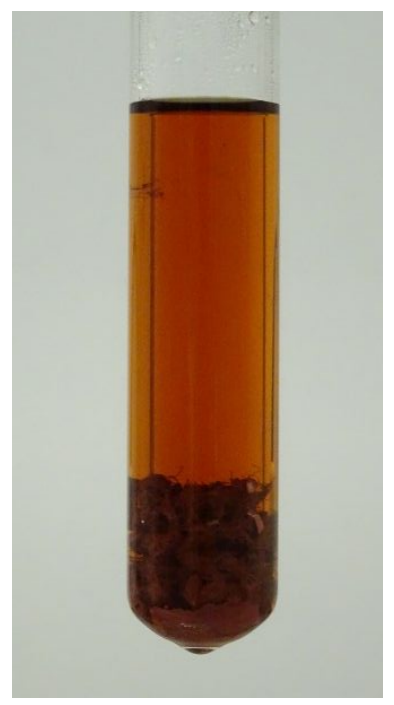

Figure S1. Digital photograph taken at the end of the synthesis featuring a reaction vessel containing reddish colored cellulose fibers suspended in a transparent, orange liquid. 


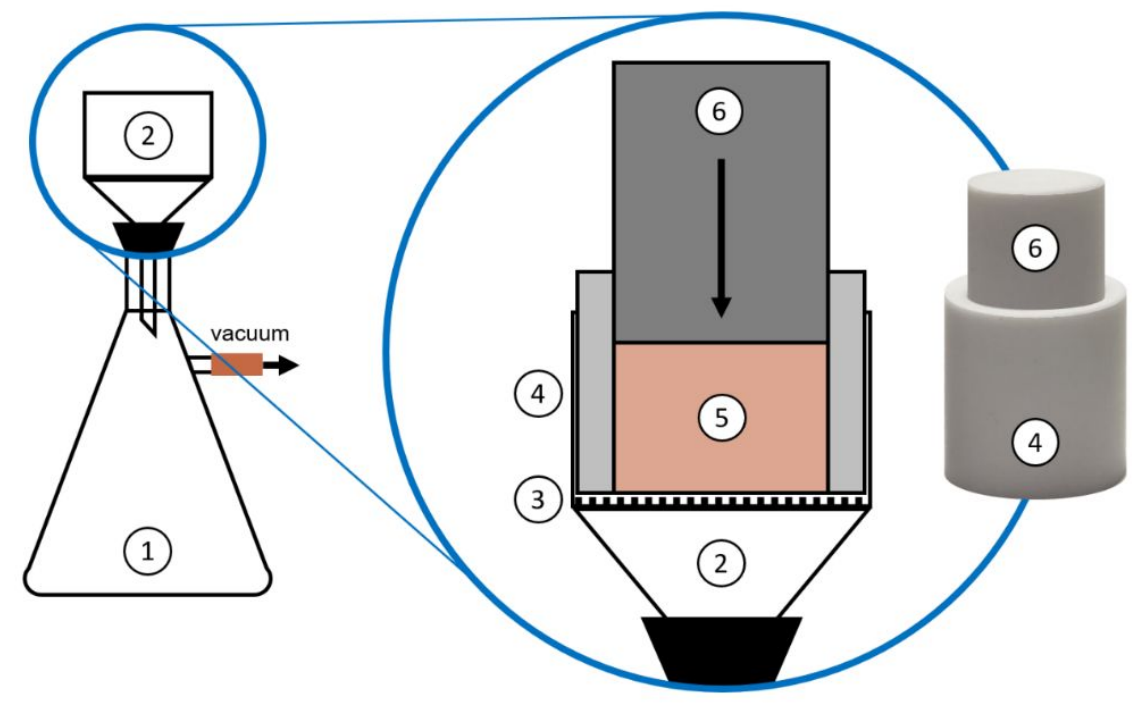

Figure S2. Setup for vacuum-filtration of copper impregnated cellulose fibers to fabricate mats: (1) Büchner flask, (2) Büchner funnel, (3) filter paper, (4) Teflon pipe with $3.0 \mathrm{~cm}$ inner diameter, (5) dispersion of copper impregnated cellulose fibers in deionized water, (6) Teflon cylinder with $3.0 \mathrm{~cm}$ outer diameter.

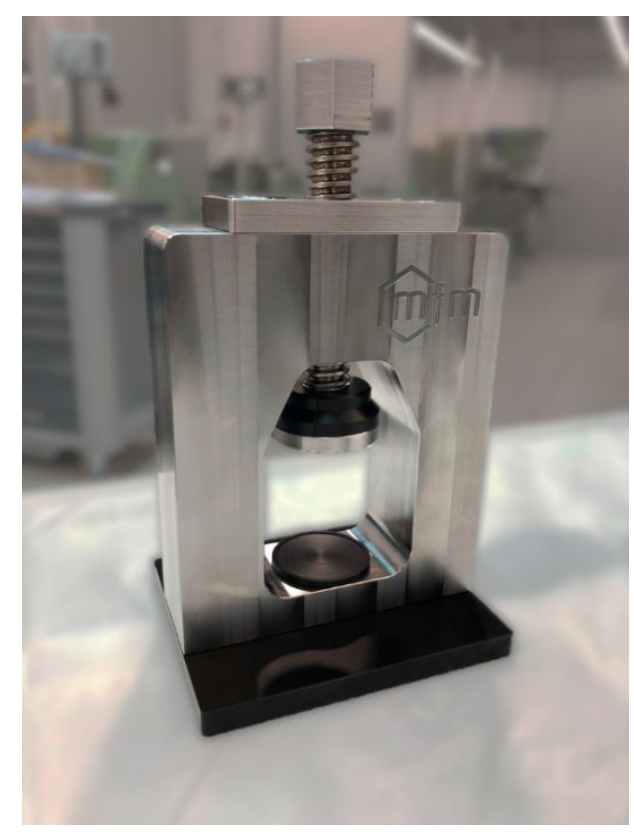

Figure S3. Digital photograph of the custom-made press for processing copper impregnated cellulose fiber mats into electrically conductive paper (mfm logo (C) Markus Niederberger) 
Table S1. Conversion of the applied torque on the press into force acting on the copper impregnated cellulose fiber mats.

\begin{tabular}{c|c} 
torque $[\mathrm{Nm}]$ & force $[\mathrm{kN}]$ \\
\hline 0 & 0 \\
5 & 2.04 \\
10 & 3.93 \\
15 & 5.51 \\
20 & 6.85 \\
25 & 8.33
\end{tabular}
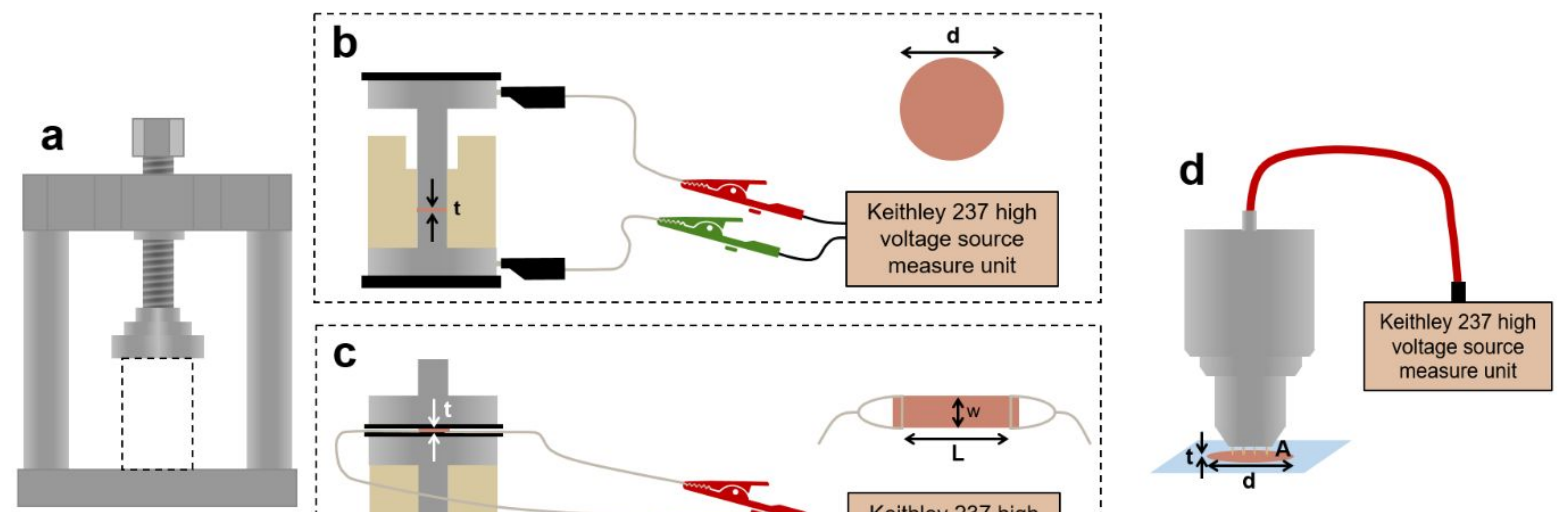

Figure S4. (a) Schematic of the custom-made press, (b) setup for resistance measurements in out-of-plane direction, (c) in in-plane direction and (d) for sheet resistance measurements. The dimensions of the coins and stripes are $d=1.2 \mathrm{~cm}, A=1.13 \mathrm{~cm}^{2}, w=0.3 \mathrm{~cm}, L=1.0 \mathrm{~cm}$, and $t$ differs depending on the applied force. 


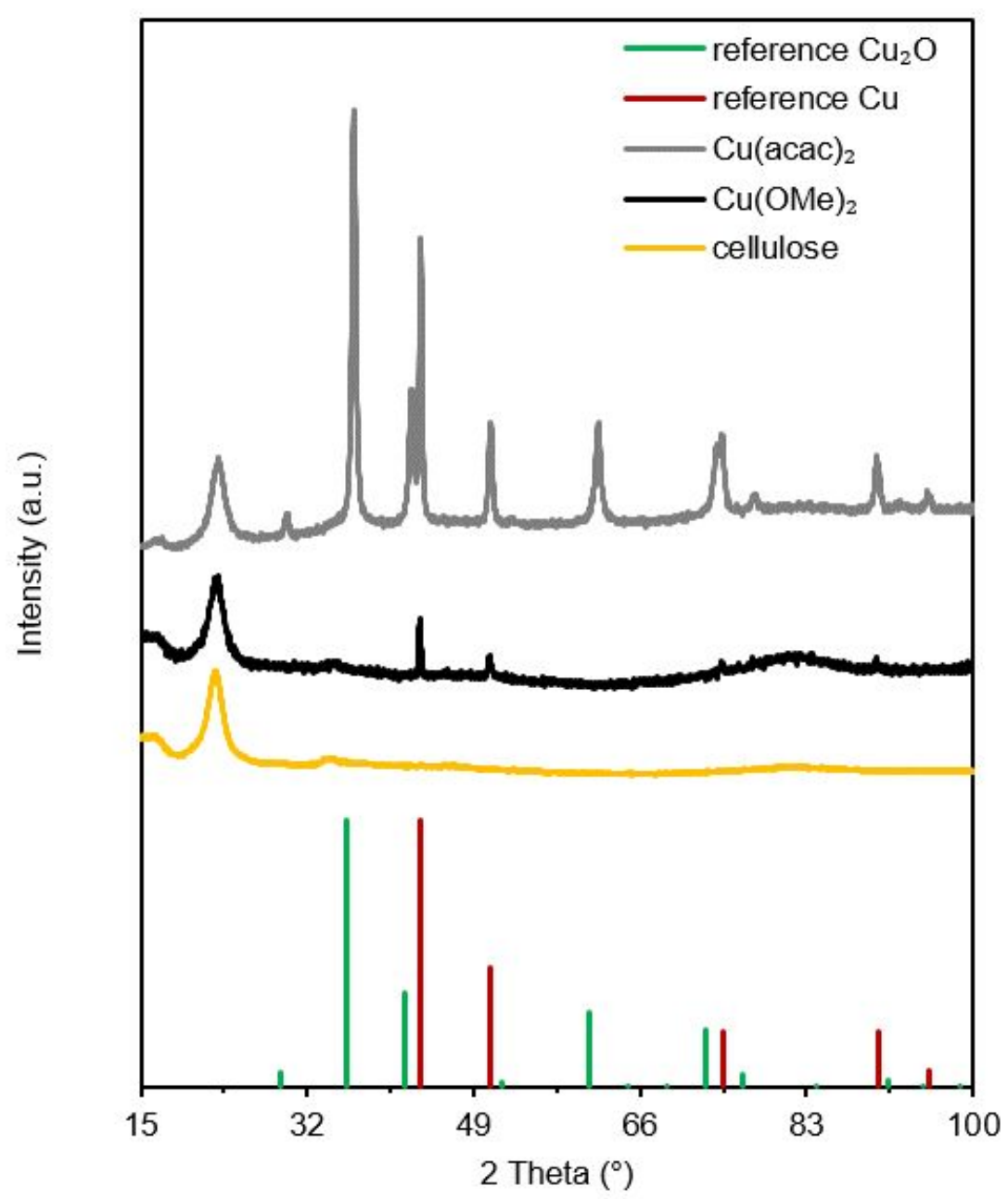

Figure S5. XRD patterns of pristine cellulose (yellow line) and of the copper impregnated cellulose fibers. The copper colloids were synthesized with $\mathrm{Cu}(\mathrm{OMe})_{2}$ (black line) or $\mathrm{Cu}(\mathrm{acac})_{2}$ (gray line). The vertical bars indicate the references of copper (red, ICDD PDF No. 04-009-2090) and copper oxide (green, ICDD PDF No. 04-018-4664). 


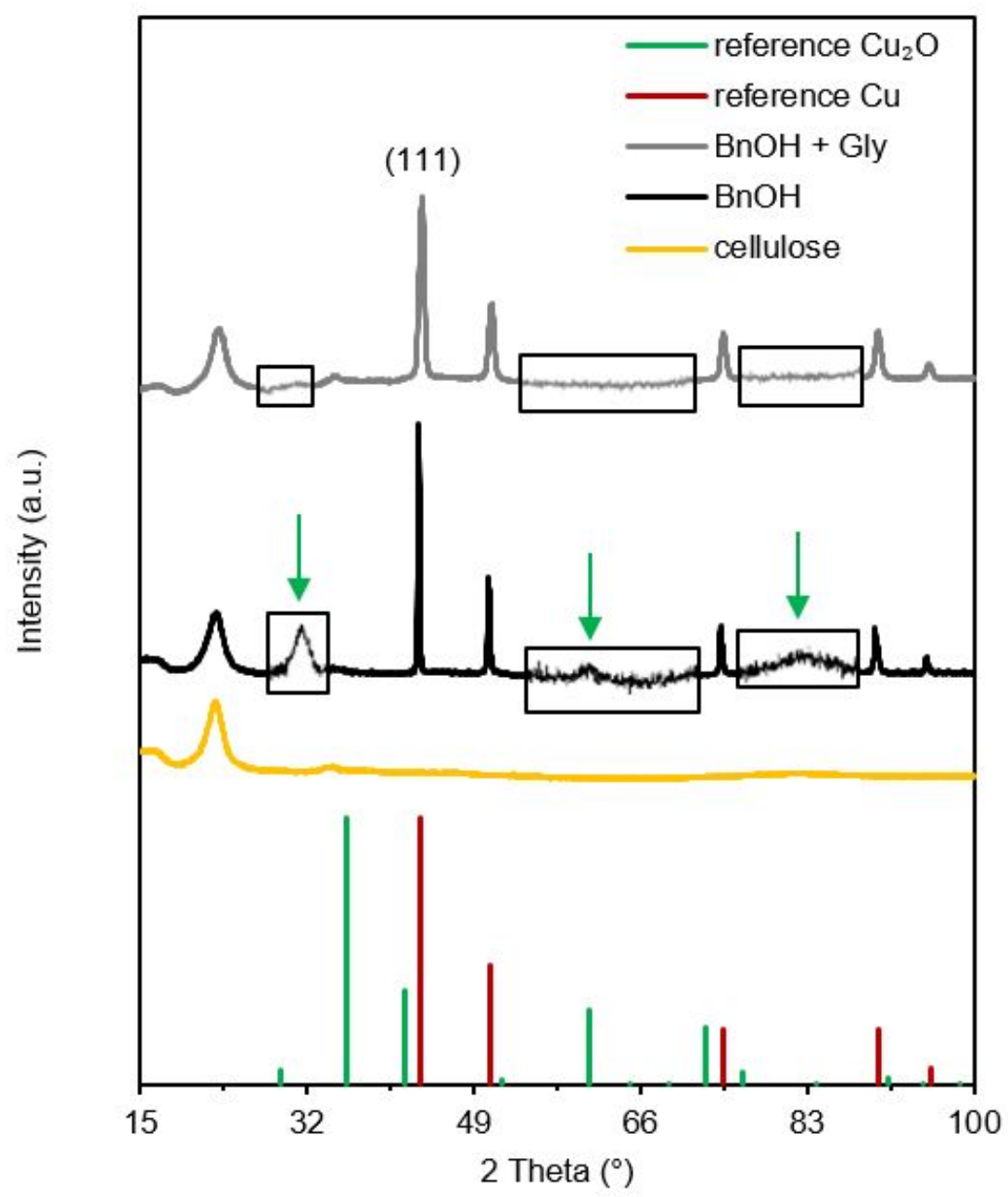

Figure S6. XRD patterns of pristine cellulose (yellow line) and of the copper impregnated cellulose fibers. The copper colloids were synthesized using benzyl alcohol $(\mathrm{BnOH}-$ black line) or benzyl alcohol plus glycerol $(\mathrm{BnOH}+\mathrm{Gly}-$ gray line). Magnified areas are marked with a black box and green arrows point to copper oxide peaks in these areas. The vertical bars indicate the references of copper (red, ICDD PDF No. 04-009-2090) and copper oxide (green, ICDD PDF No. 04-0184664). 


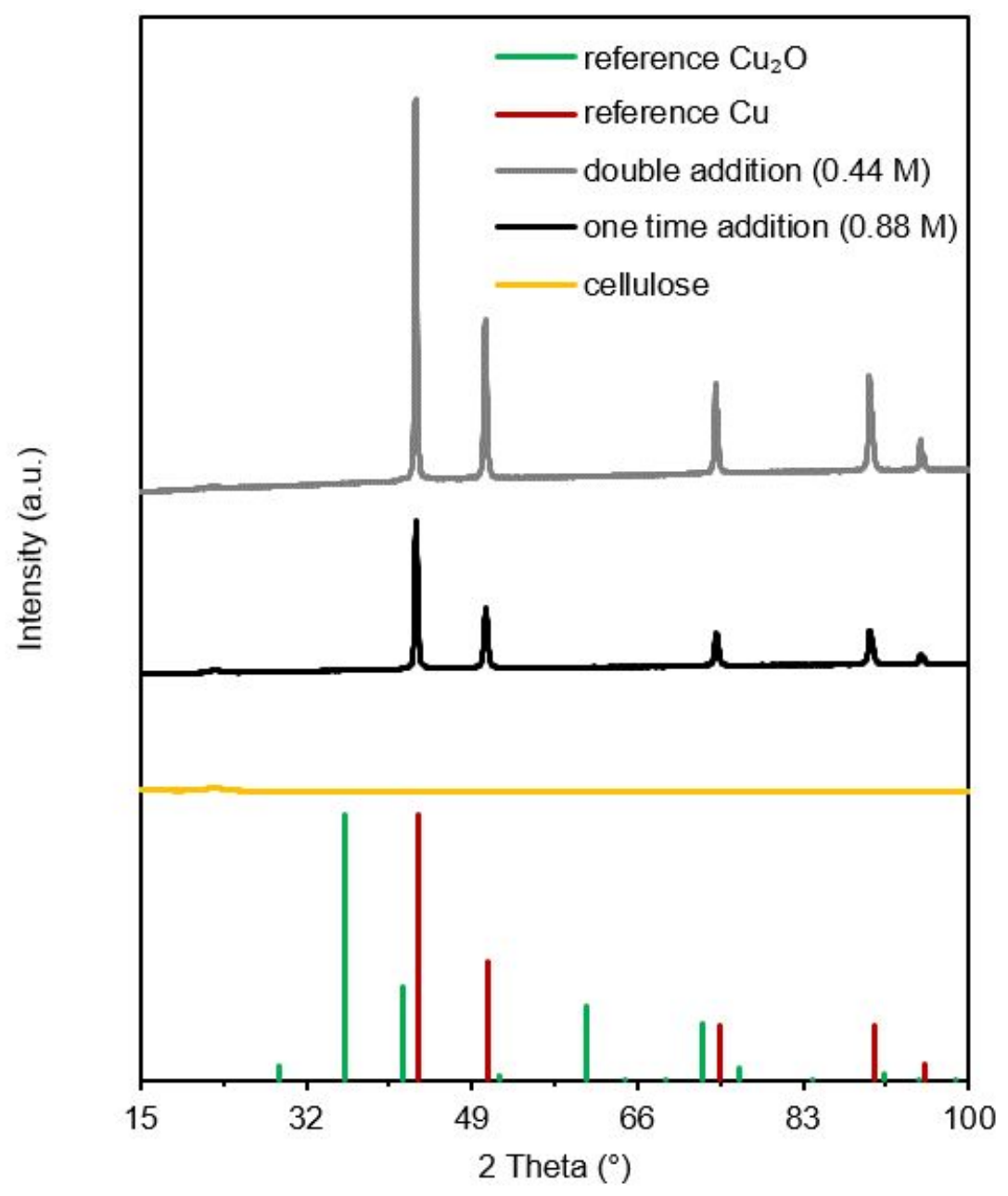

Figure S7. XRD patterns of pristine cellulose (yellow line) and of the copper impregnated cellulose fibers. The copper colloids were synthesized using a onetime addition of a $0.88 \mathrm{M}$ reactant solution (black line) or a double addition of a $0.44 \mathrm{M}$ reactant solution. The vertical bars indicate the references of copper (red, ICDD PDF No. 04-009-2090) and copper oxide (green, ICDD PDF No. 04-018-4664). 


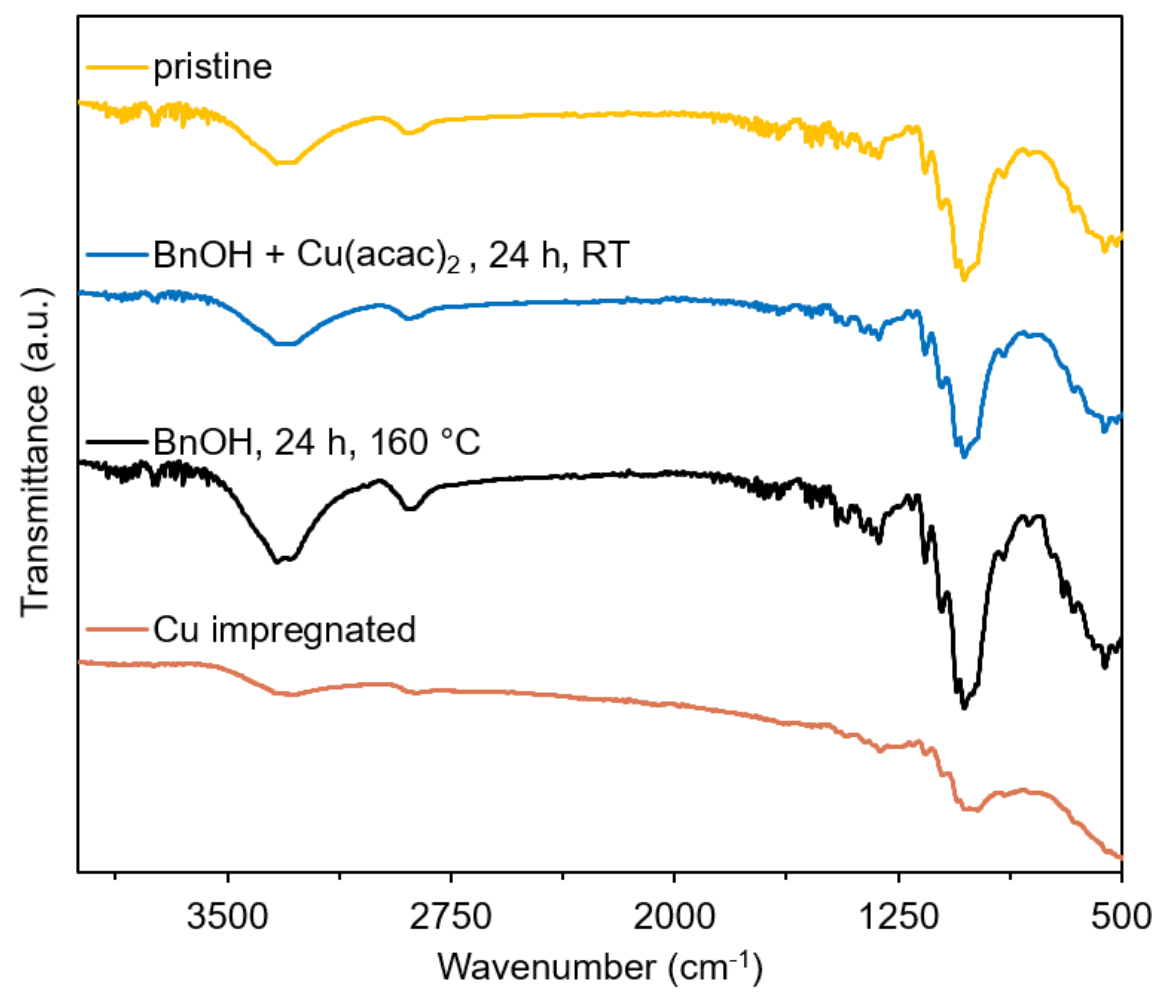

Figure S8. ATR-FTIR spectra of dried, pristine cellulose (yellow), cellulose placed in a solution of benzyl alcohol $(\mathrm{BnOH})$ and $\mathrm{Cu}(\mathrm{acac})_{2}$ at room temperature $(\mathrm{RT})$ for 24 hours (blue), cellulose treated in benzyl alcohol at $160{ }^{\circ} \mathrm{C}$ for 24 hours (black), and copper $(\mathrm{Cu})$ impregnated cellulose (orange). 

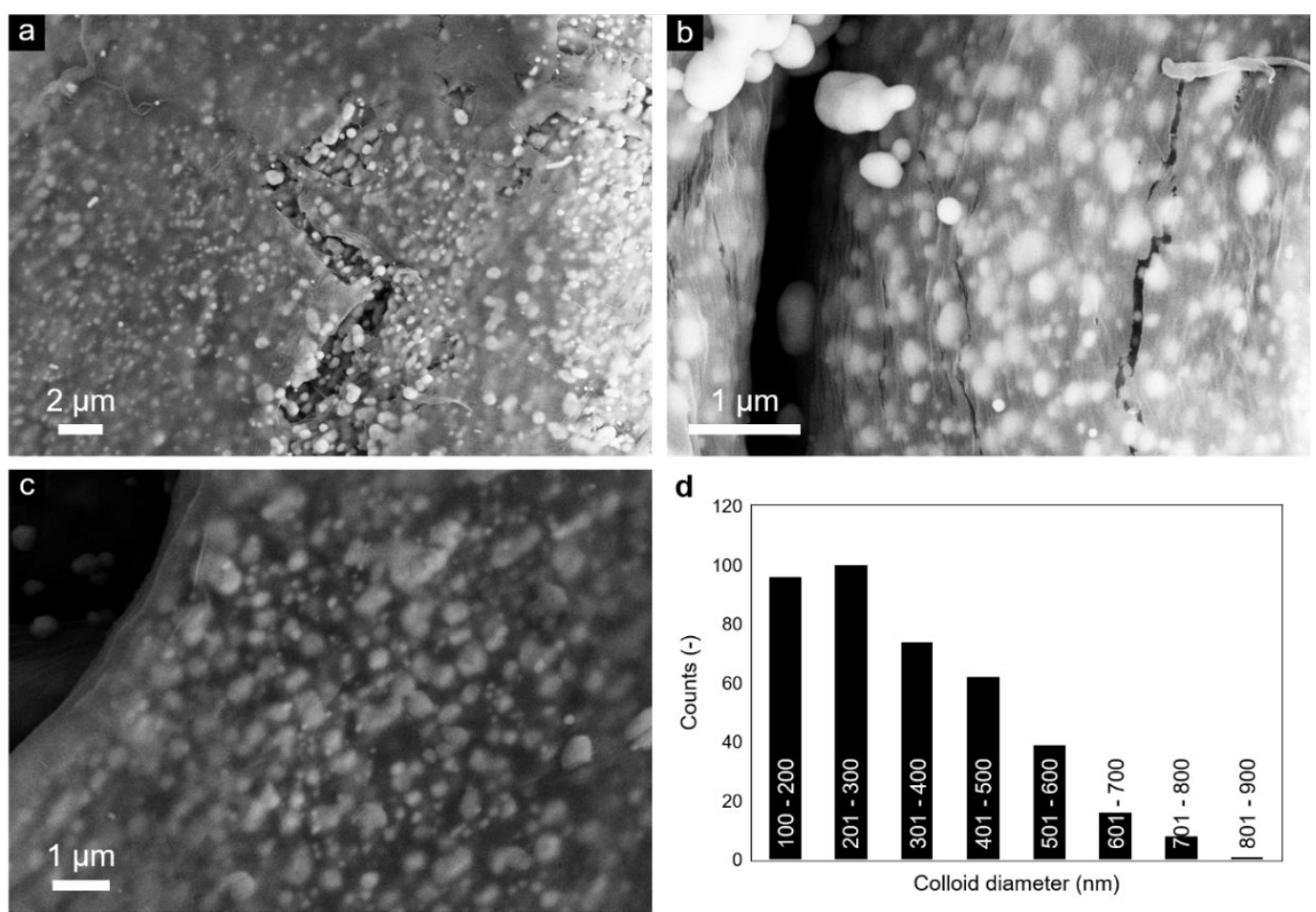

Figure S9. (a-c) SEM images of copper impregnated cellulose fibers. (d) Histogram of the copper colloid diameter distribution inside the cellulose fibers obtained from analyzing images (a), (b) and (c).

a

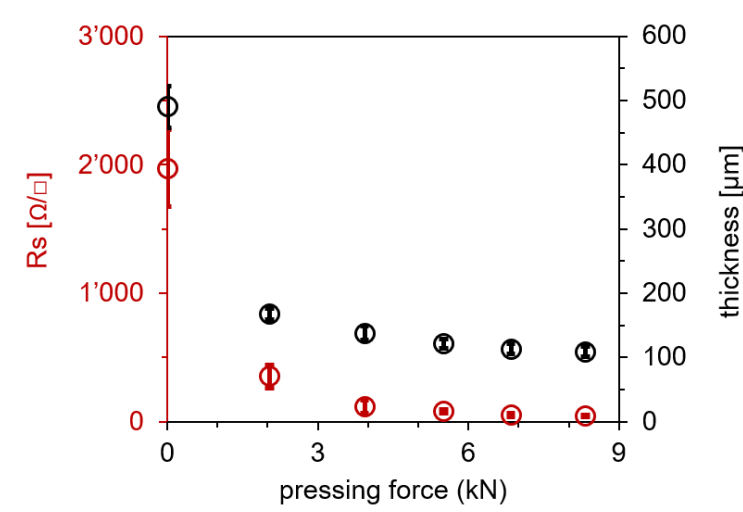

b

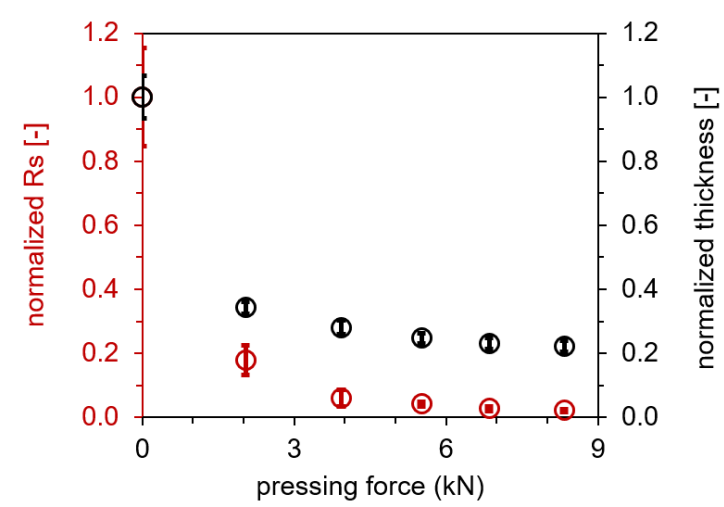

Figure S10. (a) Sheet resistance $\left(R_{\mathrm{s}}\right)$ of the copper impregnated cellulose fiber mats and papers and their thicknesses vs pressing force, (b) normalized $R_{\mathrm{s}}$ and normalized thickness of mats and papers vs pressing force, both normalized to the corresponding values of the mat at $0 \mathrm{kN}$. 

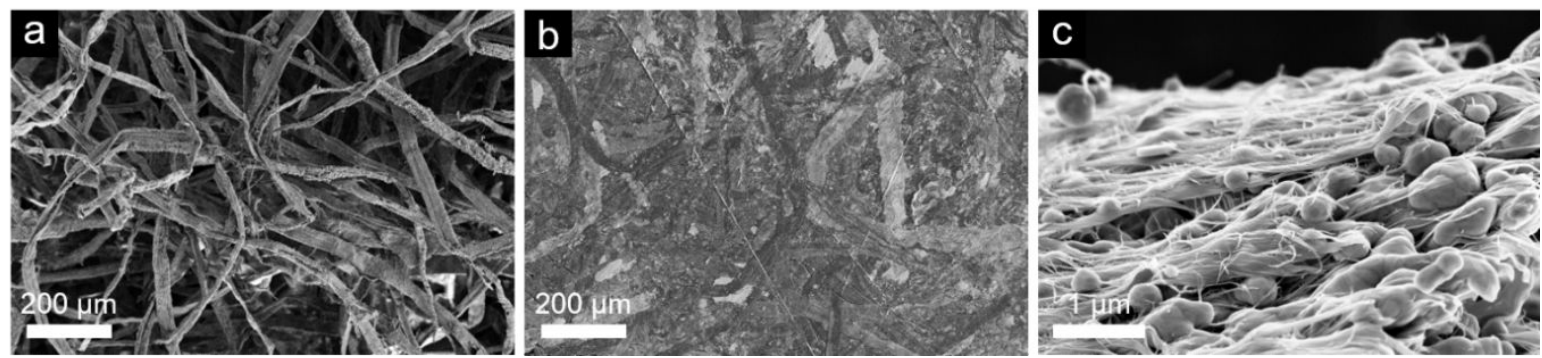

Figure S11. SEM images of (a) a mat made of randomly aligned loosely packed copper impregnated cellulose fibers, (b) a pressed copper impregnated cellulose paper, (c) the surface of an individual cellulose fiber after pressing.
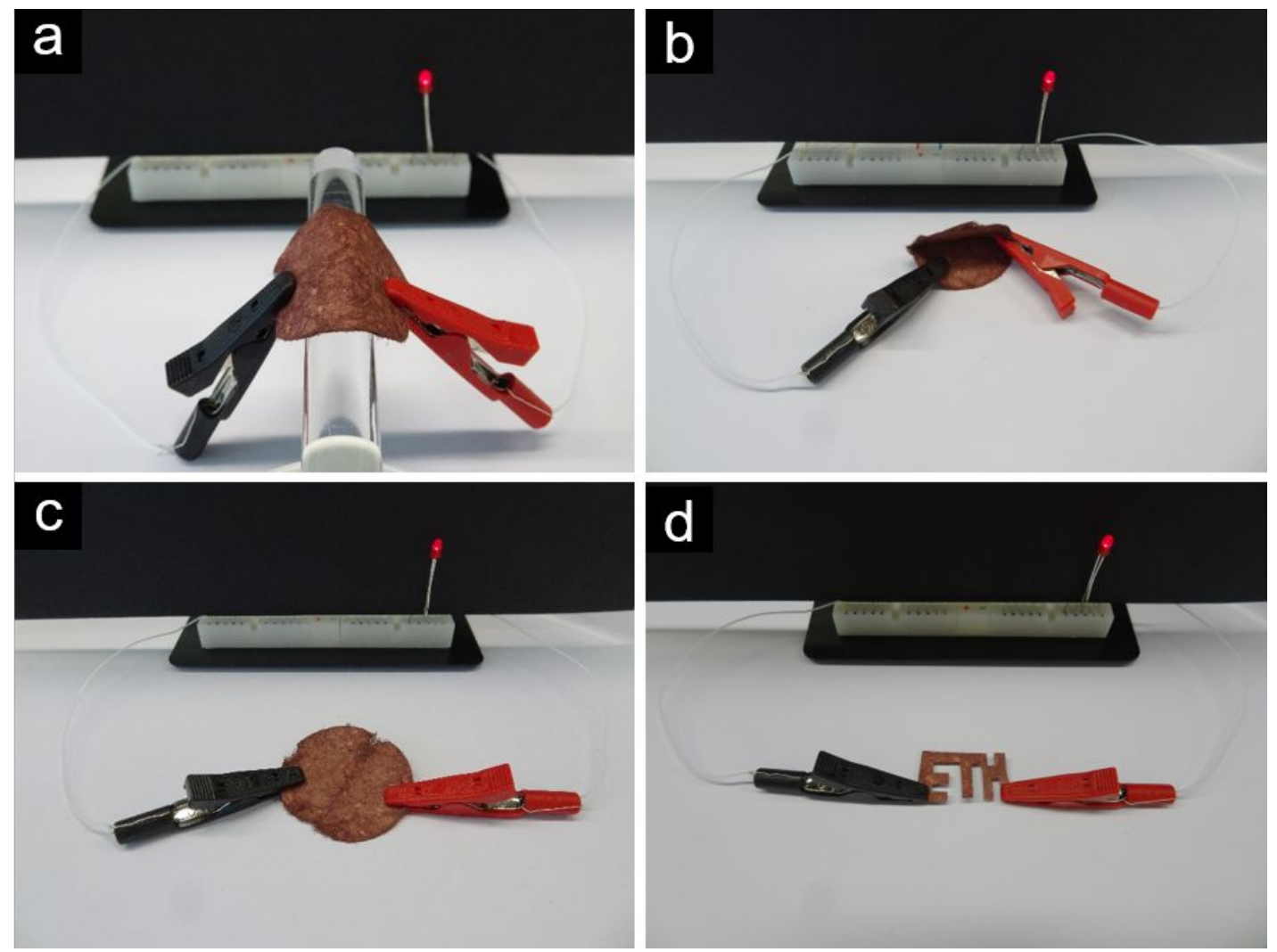

Figure S12. Digital photographs of the experimental setup to qualitatively observe the electrical conductivity of copper impregnated cellulose paper in (a) bent, (b) folded, (c) unfolded, and (d) cut form. Two crocodile clips connect the paper with a $3 \mathrm{~V}$ coin cell and a red $\operatorname{LED}(2.5 \mathrm{~V}, 25 \mathrm{~mA}, 100 \Omega)$ on a breadboard. 